\title{
Efecto de la edad relativa en los mundiales de baloncesto FIBA en cateogrías inferiores (1979-2011)
}

\author{
Relative age effect in lower categories of fiba basketball \\ world championships (1979-2011)
}

\section{Efeito da idade na no basquete FIBA mundo em baixa cateogrías (1979-2011)}

\author{
Miguel Saavedra García ${ }^{1}$, Óscar Gutiérrez Aguilar², Larissa Galatti ${ }^{3}$ y Juan J. Fernández Romero \\ 1 Universidad de A Coruña, 2 Universidad Miguel Hernández de Elche y 3 Universidade Estadual de Campinas (Brasil)
}

Resumen: El efecto de la edad relativa, en inglés, relative age effect (RAE) ha sido ampliamente estudiado en deporte, pero generalmente se centra en estudios realizados en un mismo año o en una misma temporada. El presente trabajo incluye una amplia muestra de Campeonatos del Mundo de Baloncesto FIBA que abarca desde 1979 hasta el 2011, analizando las fechas de nacimiento de 3699 jugadores de baloncesto (1975 hombres y 1724 mujeres) participantes en categorías U17, U19 y U21. Mediante la prueba de chi-cuadrado y utilizando como distribución de contraste la distribución de probabilidad uniforme corregida en función de los días de cada trimestre, se ha encontrado un efecto de la edad relativa en el baloncesto masculino y en el femenino y también agrupando ambos géneros de forma conjunta. Además el efecto de la edad relativa se presenta en todas las categorías de edad (U17, U19 y U21) tanto en el baloncesto masculino como en el femenino (excepto en U21 masculino), siendo en ambos casos más pronunciado en las competiciones en las que participan los deportistas más jóvenes (U17) y progresivamente menos acentuado hasta llegar a las competiciones donde los deportistas tienen más edad (U21).

Palabras clave: Edad cronológica, baloncesto, toma de decisión, maduración, efecto de la edad relativa.

Abstract: The relative age effect (RAE) has been widely studied in sports, however these studies are generally focused on the same academic year or sport season. A large sample of FIBA Basketball World Championship between 1979 and 2011 had been included in the current paper, analysing birth dates of 3699 basketball players (1975 men and 1724 women) participants in U17, U19 and U21 categories. Chi-square goodness-of-fit tests were used to compare the trimester of birth distribution (corrected based on the days of each trimester), it has been found a relative age effect in male and female basketball also gathering together both genders. In addition the relative age effect occurs in all age categories (U17, U19 and U21) in both men's and women's basketball (except males in U21), being more strong in both cases in the competitions in which younger athletes (U17) participate and progressively less pronounced up to competitions where athletes are older (U21)

Keywords: Chronological age, basketball, decision making, maturation, relative age effect.

Resumo: $\mathrm{O}$ efeito da idade relativa, em inglês relative age effect (RAE), tem sido amplamente estudado no desporto, mas centra-se habitualmente em estudos realizados num mesmo ano ou numa mesma época desportiva. $\mathrm{O}$ presente estudo inclui uma ampla amostra de campeonatos do mundo de basquetebol FIBA desde o ano de 1979 até 2011, analisando as idades de nascimento de 3699 jogadores de basquetebol (1975 do sexo masculino e 1724 do sexo feminino) participantes em escalóes Sub-17, Sub-19 e Sub21. Mediante a prova do qui-quadrado e utilizando como distribuiçấo de contraste a distribuiçâo de probabilidade uniforme corrigida em função dos dias de cada trimestre, encontrou-se um efeito de idade relativa no basquetebol masculino, no basquetebol feminino e também quando se agruparam ambos os géneros. Verifica-se ainda o efeito de idade relativa em todos os escalóes etários (Sub-17, Sub-19 e Sub-21) tanto no basquetebol masculino, como no basquetebol feminino (exceto em Sub-21 masculino), sendo em ambos os casos mais evidente nas competiçóes em que participam os desportistas mais jovens (Sub-17) e sendo progressivamente menos evidente até chegar às competiçôes onde os desportistas têm idade mais elevada (Sub-21). Palavras-chave: Idade cronológica, basquetebol, tomada de decisão, maturação, efeito da idade relativa.

\section{Introducción}

El efecto de la edad relativa ha sido ampliamente estudiado en deportes de equipo, sobre todo en hockey sobre hielo y en fútbol. En baloncesto existen menos estudios, centrados en ligas concretas como la española (Esteva, Drobnic, Puig-

\footnotetext{
Dirección para correspondencia [Correspondence address]: Miguel Saavedra García, Facultad de Ciencias del Deporte y la Educación Física, Universidad de A Coruña, Avd. Ernesto Che Guevara, 121, Pazos, Lians, 15179 Oleiros, A Coruña (España). E-mail: miguel.saavedra@udc.es
}

dellivol, Serratosa, y Chamorro, 2006), la alemana (Schorer, Neumann, Cobley, Tietjens, y Baker, 2011) o la francesa (Delmore, y Raspaud, 2009).

El análisis de las categorías inferiores en baloncesto ha sido abordado por distintos autores, así, Esteva, y cols. (2006) encuentran una fuerte tendencia a la selección de los jugadores nacidos en los primeros trimestres del año frente a los nacidos en los últimos trimestres. Afirman que esta tendencia se man- 
tiene hasta el baloncesto profesional, si bien, va perdiendo intensidad. Explican este hecho sugiriendo que en las primeras etapas los jugadores de baloncesto se seleccionan, o bien por su maduración avanzada, o bien por uno de sus indicadores, la altura. Delorme, Chalabaev y Raspaud (2011) complementan la información anterior estableciendo que el abandono de la práctica del baloncesto ocurre en mayor medida en los deportistas nacidos en los últimos trimestres del año y sucede con menos frecuencia en los deportistas nacidos en los primeros trimestres del año, tanto en categorías masculinas como en las femeninas. Torres-Unda y cols. (2013) indican que se seleccionan más jugadores de baloncesto nacidos en el primer semestre del año durante la adolescencia y entre los seleccionados predomina una maduración temprana, (basada en medidas antropométricas y capacidades fisiológicas, pero no en cuestiones técnicas o tácticas), encontrando que aquellos que maduran antes tienen ventaja en las características antropométricas y en los resultados de los test psicológicos. Destaca el trabajo de Saavedra, Gutiérrez, Fernández, Fernández y Eiras, In press) que encuentran un efecto de la edad relativa significativo tanto en hombres como en mujeres en los últimos campeonatos mundiales en categorías inferiores organizados por la FIBA.

La gran mayoría de los trabajos se centran en una temporada deportiva (Delorme, Chalabaev y Raspaud, 2011; Esteva y cols., 2006; Schorer, y cols., 2011; Torres-Unda y cols., 2013) o en un curso académico (Cobley, Abraham, y Baker, 2008; Roberts, Boddy, Fairclough, y Stratton, 2012) para realizar sus análisis sobre el efecto de la edad relativa. Son pocos los trabajos que analizan el efecto de la edad relativa en un período de tiempo más largo, así Addona y Yates (2010) estudian éste efecto en hockey sobre hielo (NHL) desde su origen y establecen que el RAE se detectó por primera vez en Canadá, y ocurre desde el ańo 1951. Deaner, R., Lowen, A., y Cobley, S. (2013) analizan el RAE en los drafts de la NHL desde 1980 hasta el 2006. Schorer, Wattie, y Baker, (2013) en balonmano alemán analizan el RAE entre 1993 y 2007.

El objetivo de este estudio es valorar si el efecto de la edad relativa permanece presente a lo largo de la historia de los mundiales de baloncesto FIBA en las categorías inferiores.

\section{Método}

Se ha utilizado un diseño de investigación empírico, utilizando una estrategia descriptiva de tipo observacional (Ato, López y Benavente, 2013). La muestra está compuesta por la totalidad, 3699 jugadores de baloncesto (1975 hombres y 1724 mujeres) que han disputado campeonatos del mundo en categorías inferiores organizados por la FIBA (U17, U19 y U21) entre 1979 y 2011.

Se ha recogido las variables, género, categoría, campeonato y fecha de nacimiento de la página web de la FIBA (http:// www.fiba.com/). Posteriormente se ha generado la variable trimestre, que divide las fechas de nacimiento de los jugadores en cuatro trimestres que van enero a marzo (Q1), de abril a junio (Q2), de julio a septiembre (Q3) y, de octubre a de diciembre (Q4). Del mismo modo que Doblhammer, y Vaupel (2001), Delorme, y Champley (In press), y Edgar, y O’Donoghue (2005) se consideró el número de días en cada trimestre, lo que supone una pequeña corrección a la distribución de probabilidad uniforme

$$
X_{D}^{2}=\sum_{i=1}^{4} \frac{\left(n_{i}-n \cdot d_{i}\right)^{2}}{n \cdot d_{i}}
$$

$\operatorname{con} d_{1}=\frac{31+28,25+21}{365,25}, d_{2}=\frac{30+31+30}{365,25}, d_{3}=\frac{31+31+30}{365,25}$

y $d_{4}=\frac{31+30+31}{365,25}$. Donde $n_{i}$ es la frecuencia de nacimien-

tos observada en el trimestre $i$. El tamaño de la muestra se representa por $n=\sum_{i=1}^{4} n_{i}$. Así los valores de $d_{1}$ a $d_{4}$ son 0,$247091034 ; 0,249144422 ; 0,251882272$ y 0,249144422 respectivamente.

Para determinar la existencia del efecto de la edad relativa se ha utilizado la prueba de Chi-cuadrado y se ha considerado estadísticamente significativa cuando el p-valor ha sido menor de 0,05. Además se ha calculado el tamaño del efecto como la raíz cuadrada del cociente entre $\chi^{2}$ y el número de casos.

\section{Resultados}

En la tabla 1 se observa que, de forma global, la distribución observada es distinta de la esperada tanto en categoría masculina $\left(\chi^{2}=162,02 ;\right.$ g.l. $\left.=3 ; \mathrm{p}<0,001 ; \mathrm{w}=0,286\right)$, como en la femenina $\left(\chi^{2}=110,76 ;\right.$ g.l. $\left.=3 ; \mathrm{p}<0,001 ; \mathrm{w}=0,253\right)$. En el análisis por categorías se encontró que en la competición U17 la distribución observada es diferente de la esperada en categoría masculina $\left(\chi^{2}=46,02\right.$; g.l. $\left.=3 ; \mathrm{p}<0,001 ; \mathrm{w}=0,565\right)$ y en la femenina $\left(\chi^{2}=11,68 ;\right.$ g.l. $\left.=3 ; \mathrm{p}<0,010 ; \mathrm{w}=0,285\right)$. Lo mismo ocurre en la categoría U19, tanto en categoría masculina $\left(\chi^{2}=129,547\right.$; g.l. $=3 ; \mathrm{p}<0,001 ; \mathrm{w}=0,290)$ como en la femenina $\left(\chi^{2}=93,04\right.$; g.l.=3; $\mathrm{p}<0,001 ; \mathrm{w}=0,268)$. En la competición U21 en categoría masculina no se encuentra un efecto de la edad relativa significativo $\left(\chi^{2}=5,585 ;\right.$ g.l. $\left.=3 ; \mathrm{p}=0,134 ; \mathrm{w}=0,139\right)$ pero si en la femenina $\left(\chi^{2}=9,71 ;\right.$ g.l. $\left.=3 ; \mathrm{p}<0,022 ; \mathrm{w}=0,181\right)$. 
Tabla 1. Trimestre de nacimiento de los jugadores de los mundiales de Baloncesto U17, U19 y U21.

\begin{tabular}{lccccccccc}
\hline Género & Categoría & Q1 (\%) & Q2 (\%) & Q3 (\%) & Q4 $(\%)$ & $\mathrm{n}$ & $\chi^{2}$ & Sig. & w \\
\hline Masculino & Todas & $678(34.32)$ & $543(27,49)$ & $461(32,34)$ & $293(14,83)$ & 1975 & 162,020 & $\mathbf{0 , 0 0 0 ^ { * }}$ & 0,286 \\
Femenino & Todas & $568(32,95)$ & $498(28,89)$ & $359(20,82)$ & $299(17,34)$ & 1724 & 110,759 & $\mathbf{0 , 0 0 0 ^ { * }}$ & 0,253 \\
\hline \multirow{3}{*}{ Masculino } & U21 & $85(29,41)$ & $77(26,64)$ & $68(23,53)$ & $59(20,42)$ & 289 & 5,585 & 0,134 & 0,139 \\
& U19 & $526(34,11)$ & $428(27,76)$ & $366(23,74)$ & $222(14,40)$ & 1542 & 129,547 & $\mathbf{0 , 0 0 0 ^ { * }}$ & 0,290 \\
& U17 & $67(46,53)$ & $38(26,39)$ & $27(18,75)$ & $12(8,33)$ & 144 & 46,015 & $\mathbf{0 , 0 0 0 ^ { * }}$ & 0,565 \\
\hline \multirow{3}{*}{ Femenino } & U21 & $83(28,72)$ & $86(29,76)$ & $66(22,84)$ & $54(18,69)$ & 289 & 9,721 & $\mathbf{0 , 0 2 1 ^ { * }}$ & 0,181 \\
& U19 & $435(33,69)$ & $372(28,81)$ & $262(20,29)$ & $222(17,20)$ & 1291 & 93,044 & $\mathbf{0 , 0 0 0 ^ { * }}$ & 0,268 \\
& U17 & $50(34,72)$ & $40(27,78)$ & $31(21,53)$ & $23(15,97)$ & 144 & 11,678 & $\mathbf{0 , 0 0 9 ^ { * }}$ & 0,285 \\
\hline
\end{tabular}

*Significación estadística con la prueba de Chi-cuadrado $\left(\chi^{2}\right)$.

En la tabla 2 se presenta el efecto de la edad relativa en todas las competiciones analizadas, ordenadas por género, categoría y ańo. En la categoría masculina U21 no se observa un efecto de la edad relativa significativo. En la categoría masculina U19 se aprecia un efecto significativo desde 1987 hasta el 2011, manteniéndose el tamaño del efecto en valores superiores a 0,3 en la mayor parte de las competiciones (excepto en 1987, 1995 y 2003). Es destacable que en las competiciones de 1979 y 1983 el efecto de la edad relativa no presenta significación estadística. En categoría masculina U17, el único campeonato celebrado en el año 2010 pre- senta un fuerte efecto de la edad relativa $\left(\chi^{2}=46,02\right.$; g.l. $=3$; $\mathrm{p}<0,001 ; \mathrm{w}=0,565)$.

En categoría femenina U21 no se observa significación estadística en el efecto de la edad relativa. En la categoría femenina U19 presenta un efecto significativo de manera continua desde el año 2001 al 2011, el tamaño del efecto se incrementa a lo largo del tiempo. En los años 1985, 1989 y 1997 la competición U19 femenina no presenta un efecto de la edad relativa significativo. En la competición femenina U17, el único campeonato celebrado en el año 2010 presenta un efecto de la edad relativa significativo $\left(\chi^{2}=11,68 ;\right.$ g.l. $\left.=3 ; \mathrm{p}<0,010 ; \mathrm{w}=0,285\right)$.

Tabla 2. Trimestre de nacimiento de los jugadores de los mundiales de Baloncesto por género, categoría y fecha de celebración.

\begin{tabular}{|c|c|c|c|c|c|c|c|c|c|c|}
\hline Género & Categoría & Año & Q1 & Q2 & Q3 & Q4 & $\mathrm{n}$ & $x^{2}$ & Sig. & $\mathrm{w}$ \\
\hline \multirow{2}{*}{ Masculino } & \multirow{2}{*}{ U21 } & 2005 & 43 & 42 & 30 & 31 & 146 & 4,240 & 0,237 & 0.170 \\
\hline & & 2011 & 42 & 35 & 38 & 28 & 143 & 2,995 & 0,392 & 0.145 \\
\hline \multirow{10}{*}{ Masculino } & \multirow{10}{*}{ U19 } & 1979 & 10 & 5 & 5 & 5 & 25 & 3,110 & 0,375 & 0.353 \\
\hline & & 1983 & 21 & 14 & 16 & 13 & 64 & 2,474 & 0,480 & 0.197 \\
\hline & & 1987 & 36 & 33 & 26 & 17 & 112 & 7,850 & $0,049^{*}$ & 0.265 \\
\hline & & 1991 & 64 & 59 & 50 & 18 & 191 & 27,112 & $0,000^{*}$ & 0.377 \\
\hline & & 1995 & 61 & 49 & 46 & 34 & 190 & 8,071 & $0,045^{*}$ & 0.206 \\
\hline & & 1999 & 66 & 57 & 50 & 19 & 192 & 26,375 & $0,000^{*}$ & 0.371 \\
\hline & & 2003 & 58 & 65 & 34 & 35 & 192 & 16,198 & $0,001^{*}$ & 0.290 \\
\hline & & 2007 & 67 & 50 & 50 & 25 & 192 & 19,057 & $0,000^{*}$ & 0.315 \\
\hline & & 2009 & 74 & 47 & 42 & 29 & 192 & 23,077 & $0,000^{*}$ & 0.347 \\
\hline & & 2011 & 69 & 49 & 47 & 27 & 192 & 18,883 & $0,000^{*}$ & 0.314 \\
\hline Masculino & U17 & 2010 & 67 & 38 & 27 & 12 & 144 & 46,015 & $0,000^{*}$ & 0.565 \\
\hline \multirow{2}{*}{ Femenino } & \multirow{2}{*}{$\mathrm{U} 21$} & 2003 & 45 & 39 & 33 & 28 & 145 & 4,735 & 0,192 & 0.181 \\
\hline & & 2007 & 38 & 47 & 33 & 26 & 144 & 6,616 & 0,085 & 0.214 \\
\hline \multirow{5}{*}{ Femenino } & \multirow{5}{*}{ U19 } & 1985 & 21 & 9 & 10 & 11 & 51 & 7,520 & 0,057 & 0.384 \\
\hline & & 1989 & 35 & 37 & 22 & 24 & 118 & 6,130 & 0,105 & 0.228 \\
\hline & & 1993 & 38 & 38 & 26 & 18 & 120 & 9,852 & $0,020^{*}$ & 0.287 \\
\hline & & 1997 & 39 & 40 & 35 & 26 & 140 & 3,565 & 0,312 & 0.160 \\
\hline & & 2001 & 52 & 38 & 28 & 26 & 144 & 12,283 & $0,006^{*}$ & 0.292 \\
\hline
\end{tabular}




\begin{tabular}{lllllllllcc}
\hline Género & Categoría & Año & Q1 & Q2 & Q3 & Q4 & n & $\chi^{2}$ & Sig. & w \\
\hline & & 2005 & 47 & 41 & 32 & 25 & 145 & 8,111 & $\mathbf{0 , 0 4 4 ^ { * }}$ & 0.237 \\
& 2007 & 60 & 61 & 40 & 29 & 190 & 15,935 & $\mathbf{0 , 0 0 1 *}$ & 0.290 \\
& & 2009 & 72 & 51 & 31 & 35 & 189 & 22,885 & $\mathbf{0 , 0 0 0 ^ { * }}$ & 0.348 \\
& & 2011 & 71 & 57 & 38 & 28 & 194 & 23,571 & $\mathbf{0 , 0 0 0}$ & 0.349 \\
\hline Femenino & U17 & 2010 & 50 & 40 & 31 & 23 & 144 & 11,678 & $\mathbf{0 , 0 0 9}$ & 0.285 \\
\hline
\end{tabular}

*Significación estadística con la prueba de Chi-cuadrado $\left(\chi^{2}\right)$.

\section{Discusión}

Los resultados encontrados en categoría masculina son coherentes con el efecto de la edad relativa y similares a los encontrados en el baloncesto español por Esteva $y$ cols. (2006) y que establece que el 52,9\% y $51 \%$ de los jugadores de baloncesto pertenecientes a las categorías inferiores de dos grandes clubes nacionales habían nacido en el primer trimestre. Esta cifra se rebaja hasta el 33\% en cada uno de los dos primeros trimestres en la liga alemana de baloncesto (Schorer, y cols., 2011), siendo esta cifra mucho más acorde con los resultados de la presente investigación (34,3\%). Sin embargo, los datos obtenidos por Delorme y Raspaud (2009) establecen que el porcentaje de cadetes nacidos en el primer trimestre del año era $25,4 \%$, valor claramente inferior al encontrado en el presente trabajo.

En categorías femeninas, Delorme y Raspaud (2009) obtienen un porcentaje de nacimientos de jugadoras cadetes en el primer trimestre del año de $26,10 \%$, inferior al encontrado en este estudio $(33,9 \%)$.

En los campeonatos del mundo FIBA de baloncesto Saavedra $y$ cols. (In press) se encuentra un efecto de la edad relativa significativo en las categorías U17 y U19. Este efecto desaparece en la categoría U21 en los últimos campeonatos celebrados en 2005 (masculino) y 2007 (femenino). Esto también ha sido contrastado por Hancock, Ste-Marie, y Young (2013) en hockey sobre hielo masculino y por Romann, y Fuchslocher, (2011) en fútbol femenino. Estos resultados son coherentes con los obtenidos en nuestro estudio en categoría masculina, pero en categoría femenina encontramos que el efecto de la edad relativa permanece en categorías U21.
Es interesante resaltar que se ha encontrado un importante efecto de la edad relativa en todas las categorías inferiores (U17, U19 y U21), tanto masculinas como femeninas de la historia de los campeonatos del mundo de baloncesto organizados por la FIBA. El efecto de la edad relativa no solo no disminuye a lo largo del tiempo (como ocurre en la categoría U19 masculina), sino que la categoría U19 femenina aumenta.

\section{Aplicaciones prácticas}

Existe un efecto de la edad relativa significativo a lo largo de la historia de los campeonatos del mundo de baloncesto FIBA, siendo de mayor intensidad a medida que los deportistas son más jóvenes. La selección de deportistas viene determinada por su desarrollo madurativo y por uno de sus componentes, la estatura. Es necesario que los políticos deportivos y los entrenadores de baloncesto tengan en cuenta esta situación para evitar la pérdida de talentos deportivos o el abandono del deporte por haber nacido hacia el final de año.

Es necesario establecer un sistema de competición que trate de paliar el efecto de la edad relativa, para de esta forma, otorgar igualdad de oportunidades a los deportistas. Así un sistema basado en el cambio de categoría de forma individual (cuando los deportistas cumplan la edad preceptiva), un sistema de categorías por peso o por altura o una selección por cuotas, donde se obligue a seleccionar deportistas de los cuatro trimestres del año por igual podrían suponer un gran avance en este sentido.

\section{Referencias}

1. Addona, V., y Yates, P.A. (2010). A Closer Look at the Relative Age Effect in the National Hockey League. Journal of Quantitative Analysis in Sports, 6(4), Article 9.

2. Ato, M., López, J.J, y Benavente, A. (2013). Un sistema de clasificación de los diseños de investigación en psicología. Anales de psicología, 29(3), 1038-1059.

3. Cobley, S., Abraham, C., y Baker, J. (2008). Relative age effects on physical education attainment and school sport representation. Physical Education and Sport Pedagogy, 13(3), 267-276.

4. Deaner, R., Lowen, A., y Cobley, S. (2013). Born at the Wrong Time: Selection Bias in the NHL Draft. PLoS One, 8(2), e57753.

5. Delorme, N., Chalabaev, A., y Raspaud, M. (2011). Relative age is as- sociated with sport dropout: evidence from youth categories of French basketball. Scandinavian Journal of Medicine \& Science in Sports, 21(1), 120-128.

6. Delorme, N., y Champley, S. (In press). Relative Age Effect and chisquared statistics. International Review for the Sociology of Sport. Recuperado de: DOI: 10.1177/1012690213493104.

7. Delmore, N., y Raspaud, M. (2009). The relative age effect in young French basketball players: a study on the whole population. Scandinavian Journal of Medicine \& Science in Sports, 19, 235-242.

8. Doblhammer, G., y Vaupel, J.W. (2001). Lifespan depends on month of birth. Proceedings of the National Academy of Sciences, 98, $2934-$ 2939. 
9. Edgar, S., y O'Donoghue, P. (2005). Season of birth distribution of elite tennis players. Journal of Sport Sciences, 23, 1013-1020.

10. Esteva, S., Drobnic, F., Puigdellivol, J., Serratosa, L., y Chamorro, M. (2006). Fecha de nacimiento y éxito en el baloncesto profesional. $\mathrm{Me}$ dicina de l'esport, 41(149), 25-30.

11. Hancock, D.J., Ste-Marie, D.M., y Young, B.W. (2013). Coach Selections and the Relative Age Effect in Male Youth Ice Hockey. Research Quarterly for Exercise and Sport, 84, 126-130.

12. Roberts, S.J., Boddy, L.M., Fairclough, S.J., y Stratton, G. (2012). The Influence of Relative Age Effects on the Cardiorespiratory Fitness Levels of Children Age 9 to 10 and 11 to 12 Years of Age. Pediatric Exercise Science, 24, 72-83.

13. Romann, M., y Fuchslocher, J. (2011). Influence of the Selection Level, Age and Playing Position on Relative Age Effects in Swiss Women's Soccer. Talent Development \& Excellence, 3(2), 239-247.
14. Saavedra, M., Gutiérrez, O., Fernández, J.J., Fernández, D., y Eiras, G. (In press). Relative age effect in lower categories of international basketball. International Review for the Sociology of Sport, published online 30 October 2012. DOI: 10.1177/1012690212462832.

15. Schorer, J., Neumann, J., Cobley, S., Tietjens, M., y Baker, J. (2011). Lingering effects of relative age in basketball players post athletic career. International Journal of Sport Sciences \& Coaching, 6(1), 143-147.

16. Schorer, J., Wattie, N., y Baker, J. (2013). A new dimension to relative age effects: constant year effects in german youth handball. PLoS One, 8(4): e60336.

17. Torres-Unda, J., Zarrazquin, I., Gil, J., Ruíz, F., Irazusta, A., Kortajarena, M., Seco, J., y Irazusta, J. (2013). Anthropometric, physiological and maturational characteristics in selected elite and non-elite male adolescent basketball players. Journal of Sports Sciences, 31(2), 196-2 